\title{
TCHEBYCHEFF APPROXIMATIONS BY FUNCTIONS UNISOLVENT OF VARIABLE DEGREE
}

\author{
BY
}

JOHN R. RICE(')

1. The first steps toward a theory of nonlinear Tchebycheff approximations were made by T. S. Motzkin [2] and L. Tornheim [4]. They introduced the properties of unisolvence and the equivalent concept of $n$-parameter families. For approximating functions which have these properties an elegant theory of Tchebycheff approximations may be developed. Unfortunately, this class of functions does not contain any well-known functions, except for the degenerate case of linear approximating functions and transformations thereof. Motzkin [3] has shown, however, that essentially nonlinear unisolvent functions do exist.

In this paper a property weaker than unisolvence will be introduced which also allows the development of an elegant theory of Tchebycheff approximations. The class of functions possessing this property contains many elementary nonlinear approximating functions. The main definitions are given in $\S 2$. Existence and uniqueness are discussed in $\S 3$ and a theorem on the characterization of best Tchebycheff approximations is given. Also given is an interesting theorem on a topological property of the parameter space. The final section contains some examples which illustrate the ideas of the paper.

2. Euclidean $n$-dimensional space is denoted by $E_{n}$; points in $E_{n}$ are denoted by $a, b$, etc. and the coordinates of $a$ are $\left(a^{1}, a^{2}, \cdots, a^{n}\right)$. Curly brackets, \{\} , denote a set and $\{x \mid \ldots\}$ is read as "the set of $x$ such that ...." All maxima and minima are taken over $x \in[0,1]$ unless otherwise stated.

The real function $F=F(a, x)$ is defined for $x \in[0,1]$ and $a \in P$ where $P$ is a nonvoid subset of $E_{n} . F$ is continuous in the sense that given $a_{0} \in P$, $x_{0} \in[0,1]$ and $\epsilon>0$ there is a $\delta>0$ such that $a \in P, x \in[0,1],\left|a_{0}-a\right|$ $+\left|x_{0}-x\right|<\delta$ implies that $\left|F\left(a_{0}, x_{0}\right)-F(a, x)\right|<\epsilon . f(x)$ will denote a continuous function on $[0,1]$.

The Tchebycheff Approximation Problem for $f(x)$ may be stated as: Determine $a^{*} \in P$ such that $\max \left|F\left(a^{*}, x\right)-f(x)\right| \leqq \max |F(a, x)-f(x)|$ for all $a \in P$. A solution, $F\left(a^{*}, x\right)$, to the approximation problem is called a best approximation to $f(x)$.

This paper has been submitted to and accepted for publication by the Proceedings of the American Mathematical Society. It has been transferred to these Transactions, with the consent of the author, for technical reasons. Presented to the Society, October 31, 1959; received by the editors January 25, 1960 and, in revised form, June 10, 1960.

(1) Part of these results are implicitly and explicitly contained in the third chapter of the author's Ph.D. thesis at the California Institute of Technology and part of them were obtained while the author was an NRC Research Associate at the National Bureau of Standards. 
Definition 1. $F$ is solvent of degree $m$ at $a^{*} \in P$ if given a set $\left\{x_{j} \mid 0 \leqq x_{1}<x_{2}<\cdots<x_{m} \leqq 1\right\}$ and $\epsilon>0$ then there is a $\delta\left(a^{*}, \epsilon, x_{1}, \cdots, x_{m}\right)$ $>0$ such that $\left|y_{j}-F\left(a^{*}, x_{j}\right)\right|<\delta$ implies that there is a solution, $a \in P$, to $F\left(a, x_{j}\right)=y_{j}, j=1,2, \cdots, m$ with $\max \left|F(a, x)-F\left(a^{*}, x\right)\right|<\epsilon$.

Definition 2. $F$ has property $Z$ of degree $m$ at $a^{*} \in P$ if for any $a \neq a^{*}$, $F\left(a^{*}, x\right)-F(a, x)$ has at most $m-1$ zeros. It is understood that $m \geqq 1$.

Definition 3. $F$ is unisolvent of degree $m$ at $a^{*} \in P$ if (i) $F$ has property $\mathrm{Z}$ of degree $m$ at $a^{*}$ and $F$ is solvent of degree $m$ at $a^{*}$; (ii) $F$ is not solvent of degree $m+1$ at $a^{*}$.

Definition 4. $\max |F(a, x)-f(x)|$ is said to alternate $k$ times if there are $k+1$ points $0 \leqq x_{1}<x_{2}<\cdots<x_{k+1} \leqq 1$ such that $F\left(a, x_{j}\right)-f\left(x_{j}\right)$ $=-\left[F\left(a, x_{j+1}\right)-f\left(x_{j+1}\right)\right]= \pm \max |F(a, x)-f(x)|$.

3. The existence of best approximations by functions which are unisolvent of variable degree now becomes a separate question. Unisolvence of variable degree is not sufficient to guarantee the existence of best approximations. An additional assumption is needed which will assure the existence of a limiting function for a pointwise convergent sequence $\left\{F\left(a_{i}, x\right)\right\}$. In the work of Motzkin and Tornheim this has been implied by their definitions.

The uniqueness of best approximations, if they exist, is a very simple matter. It can be shown that best approximations by a function unisolvent of variable degree are unique by exactly the same argument as used for polynomials, once Theorem 1 is established.

A zero $x_{0}$ of $f(x)$ is said to be a simple zero if $f(x)$ changes sign at $x_{0}$ and a double zero if $f(x)$ does not change sign at $x_{0}$.

Lemma. Let $F$ be unisolvent of degree $n$ at $a^{*}$. Then $F(a, x)-F\left(a^{*}, x\right)$ cannot have more than $n-1$ zeros counting multiplicity.

Proof. Let $\left\{x_{j} \mid j=1,2, \cdots, k\right\}$ be the set of zeros of $F(a, x)-F\left(a^{*}, x\right)$. The degree of unisolvence of $F$ at $a$ is then at least $k+1$. Assume, for concreteness, that $x_{1}$ is a double zero and $F(a, x)-F\left(a^{*}, x\right)>0$ near $x_{1}$. Since $F$ is unisolvent of degree $k+1$ (at least) there is an $a_{1} \in P$ such that $F\left(a_{1}, x_{j}\right)$ $=F\left(a, x_{j}\right), j \neq 1 ; F\left(a_{1}, x_{1}\right)<F\left(a, x_{1}\right)$ and $\max \left|F\left(a_{1}, x\right)-F(a, x)\right|<\epsilon$. For $\epsilon$ sufficiently small it is clear that (i) every double zero of $F(a, x)-F\left(a^{*}, x\right)$ is either a double zero of $F\left(a_{1}, x\right)-F\left(a^{*}, x\right)$ or has been replaced by two zeros, (ii) $\left\{x_{j} \mid j=2,3, \cdots, k\right\}$ are zeros of $F\left(a_{1}, x\right)-F\left(a^{*}, x\right)$ and (iii) $F\left(a_{1}, x\right)$ $-F\left(a^{*}, x\right)$ has two simple zeros near $x_{1}$. Hence, counting multiplicities, the number of zeros of $F\left(a_{1}, x\right)-F\left(a^{*}, x\right)$ is greater than or equal to the number of zeros of $F(a, x)-F\left(a^{*}, x\right)$. This process is continued to obtain $a_{m} \in P$ such that $F\left(a_{m}, x\right)-F\left(a^{*}, x\right)$ has at least as many zeros as $F(a, x)-F\left(a^{*}, x\right)$ and the zeros of $F\left(a_{m}, x\right)-F\left(a^{*}, x\right)$ are all simple. This completes the proof.

The theorem characterizing best approximations appears in a slightly different form and is stated as follows: 
Theorem 1. Let $F$ be unisolvent of variable degree in $P$, and let $m\left(a^{*}\right)$ be the degree of unisolvence at $a^{*}$. Then $F\left(a^{*}, x\right)$ is the best approximation to $f(x)$ if and only if $\max \left|F\left(a^{*}, x\right)-f(x)\right|$ alternates $m\left(a^{*}\right)$ times.

Proof. Assume that $\max F\left(a^{*}, x\right)-f(x)$ alternates $m\left(a^{*}\right)$ times. Then the assumption that there is an $a \in P$ such that $\max |F(a, x)-f(x)|$ $<\max \left|F\left(a^{*}, x\right)-f(x)\right|$ will lead to a contradiction. For examine the value of $F\left(a, x_{j}\right)-F\left(a^{*}, x_{j}\right)=F\left(a, x_{j}\right)-f\left(x_{j}\right)-F\left(a^{*}, x_{j}\right)+f\left(x_{j}\right)$ at the $n+1$ points where $\max \left|F\left(a^{*}, x\right)-f(x)\right|$ alternates. It is seen that $\operatorname{sgn}\left[F\left(a, x_{j}\right)-F\left(a^{*}, x_{j}\right)\right]$ $=-\operatorname{sgn}\left[F\left(a, x_{j+1}\right)-F\left(a^{*}, x_{j+1}\right)\right], j=1,2, \cdots, m\left(a^{*}\right)+1$. Hence $F(a, x)$ $-F\left(a^{*}, x\right)$ has at least $m\left(a^{*}\right)$ zeros which is a contradiction.

Assume that $\max \left|F\left(a^{*}, x\right)-f(x)\right|$ alternates exactly $k$ times, $k<n$, $n=m\left(a^{*}\right)$. It will be shown that $F\left(a^{*}, x\right)$ cannot be a best approximation to $f(x)$.

If $\max \left|F\left(a^{*}, x\right)-f(x)\right|$ is not assumed at both 0 and 1 then one of the intervals $[0, \delta],[1-\delta, 1]$, say $[0, \delta]$ for concreteness, is chosen with $\delta$ determined so that for $x \in[0, \delta]$ and some $\epsilon_{0}>0$, we have $\left|F\left(a^{*}, x\right)-f(x)\right|$ $<\max \left|F\left(a^{*}, x\right)-f(x)\right|-\epsilon_{0}$. Let $0=x_{0}<x_{n-k}<x_{n-k+1}<\cdots<x_{n}=1$ divide $[0,1]$ into $k+1$ subintervals so that (i) $F\left(a^{*}, x_{j}\right)-f\left(x_{j}\right)=0, j=n-k, n-k+1$, ..., n-1; (ii) $\max \left|F\left(a^{*}, x\right)-f(x)\right|$ alternates exactly once in any two adjacent subintervals but does not alternate in any one subinterval. Choose $n-k-1$ distinct points, $\left\{x_{j} \mid j=1,2, \cdots, n-k-1, x_{j}<x_{j+1}\right\}$, in $[0, \delta]$. Let $\alpha_{j}=\max \left(F\left(a^{*}, x\right)-f(x)\right)-\min \left(F\left(a^{*}, x\right)-f(x)\right)$ with the maximum and minimum taken over $\left[x_{j}, x_{j+1}\right], j=n-k-1, n-k, \cdots, n-1$. Let $\eta_{j}=\max \left|F\left(a^{*}, x\right)-f(x)\right|-\alpha_{j} / 2, j=n-k-1, n-k, \cdots, n-1$ and $\epsilon=\min \left(\eta_{n-k-1}, \cdots, \quad \eta_{n-1}, \epsilon_{0} / 2\right)$. Let $x^{\prime}$ denote a point where $\max \left|F\left(a^{*}, x\right)-f(x)\right|$ is attained.

Since $F$ is solvent at $a^{*}$ there is an $a \in P$ such that $F(a, x)-F\left(a^{*}, x\right)$ changes sign at $x_{j}, j=1,2, \cdots, n-1$ and $\left|F\left(a, x^{\prime}\right)-f\left(x^{\prime}\right)\right|<\left|F\left(a^{*}, x^{\prime}\right)-f\left(x^{\prime}\right)\right|$ with $\max \left|F(a, x)-F\left(a^{*}, x\right)\right|<\epsilon$. Since $F(a, x)-F\left(a^{*}, x\right)$ cannot have any more zeros, $F(a, x)$ is uniformly closer to $f(x)$ than $F\left(a^{*}, x\right)$ except in $[0, \delta]$. Therefore $F(a, x)$ is a better approximation than $F\left(a^{*}, x\right)$ to $f(x)$.

If $\max \left|F\left(a^{*}, x\right)-f(x)\right|$ is assumed at both 0 and 1 let the interval $[0, \delta]$ be replaced by $\left[x_{n-k}-\delta, x_{n-k}\right]$ where $\left\{x_{j} \mid j=0, n-k, \cdots, n\right\}, \delta, \epsilon_{0}, \alpha_{j}, \eta_{j}$ and $\epsilon$ are defined in a manner analogous to the preceding construction.

For $n-k-1$ even choose $n-k-1$ points

$$
\left\{x_{j} \mid x_{n-k}-\delta \leqq x_{1}<x_{2}<\cdots<x_{n-k-1}<x_{n-k}\right\}
$$

and determine $a \in P$ so that $|F(a, 0)-f(0)|<\left|F\left(a^{*}, 0\right)-f(0)\right|,|F(a, 1)-f(1)|$ $<\left|F\left(a^{*}, 1\right)-f(1)\right|,\left|F(a, x)-F\left(a^{*}, x\right)\right|<\epsilon$ and $F(a, x)-F\left(a^{*}, x\right)$ changes sign at the $x_{j}, j=1,2, \cdots, n-1 . F(a, x)$ is a better approximation than $F\left(a^{*}, x\right)$ to $f(x)$.

For $n-k-2$ even choose $n-k-2$ points 


$$
\left\{x_{j} \mid x_{n-k}-\delta \leqq x_{2}<x_{3}<\cdots<x_{n-k-1}<x_{n-k}\right\}
$$

and determine $a$ so that $|F(a, 0)-f(0)|<\left|F\left(a^{*}, 0\right)-f(0)\right|,|F(a, 1)-f(1)|$ $<\left|F\left(a^{*}, 1\right)-f(1)\right|,\left|F(a, x)-f\left(a^{*}, x\right)\right|<\epsilon$ for $x \in[0,1]$ and $F(a, x)-F\left(a^{*}, x\right)$ changes sign at the $x_{j}, j=2,3, \cdots, n-1 . F(a, x)-F\left(a^{*}, x\right)$ cannot have another zero without having two more, counting multiplicity, and hence $F(a, x)$ is a better approximation than $F\left(a^{*}, x\right)$ to $f(x)$. This concludes the proof.

The following theorem points out an interesting topological property of the parameter set of functions unisolvent of variable degree.

Theorem 2. Let $F$ be unisolvent of variable degree $m(a)$ for $a \in P$. Define $P_{k}=\{a \mid a \in P, m(a) \geqq k\}$. Then $P_{k}$ is an open set.

Proof. Let $a^{*} \in P_{k}$ be given. Then the degree of solvence of $F$ at $a^{*}$ is $k$ and there is an $a_{1} \in P$ such that $F\left(a^{*}, x\right)-F\left(a_{1}, x\right)$ has $k+1$ simple zeros in the interior of $[0,1]$. Let $\left\{x_{j} \mid j=1,2, \cdots, k-1 ; x_{j}<x_{j+1}\right\}$ be the set of these zeros and set $x_{0}=0, x_{k}=1$. Let

$$
\delta=\min _{j} \max _{x \in\left[x_{j}, x_{j+1}\right]}\left|F\left(a^{*}, x\right)-F\left(a_{1}, x\right)\right|, \quad j=0,1,2, \cdots, k-1 .
$$

It is clear that for any $a \in P$ if $\max \left|F(a, x)-F\left(a^{*}, x\right)\right|<\delta$ then $F(a, x)$ $-F\left(a_{1}, x\right)$ has at least $k-1$ zeros.

Since $F$ is continuous there is a neighborhood of $a^{*}, N\left(a^{*}\right)$, such that if $a \in N\left(a^{*}\right)$ then $\max \left|F(a, x)-F\left(a^{*}, x\right)\right|<\delta$ and $F(a, x)-F\left(a_{1}, x\right)$ has at least $k-1$ zeros. Hence the degree of unisolvence of $F$ at $a$ is at least $k$.

4. In this section three examples of functions which are unisolvent of variable degree are given. Each of these examples is a simple function. The nature of the variation of degree of unisolvence is quite different for each of these examples.

The first and most interesting example of this theory is approximation by rational functions. Here $F$ is of the form

$$
s(x)\left(\sum_{k=0}^{n} a^{k+1} x^{k}\right) /\left(\sum_{k=n+1}^{n+m+1} a^{k+1} x^{k-n-1}\right) \text { where } s(x)>0
$$

and is continuous on $[0,1] . F$ is normalized by the condition $\sum_{k=1}^{n+m+2}\left(a^{k}\right)^{2}=1$. $P$ is then taken to be the subset of the unit sphere in $E_{n+m+2}$ for which $\sum_{k=n+1}^{n+m+2} a^{k} x^{k-n-1} \neq 0$ for $x \in[0,1]$.

The classical theorem characterizing best approximations is [1]:

" $R(a, x)=s(x)\left(a^{n-\alpha} x^{n-\alpha}+\cdots+a^{0}\right) /\left(b^{m-\beta} x^{m-\beta}+\cdots+b^{0}\right)$ (in reduced terms) where $0 \leqq \alpha \leqq n, 0 \leqq \beta \leqq m, b^{m-\beta} \neq 0, a^{n-\alpha} \neq 0, b^{0} \neq 0$ is the best approximation to $f(x)$ if and only if $\max |R(a, x)-f(x)|$ alternates at least $m+n$ $+1-d$ times where $d=\min (\alpha, \beta)$; if $R(a, x)=0$ then $d=m$." In general $R(a, x)$ is unisolvent of degree $m+n+1$, but at points of $P$ where the numerator and 
denominator contain common factors then there is an effective loss of parameters and the degree of unisolvence is decreased at such points. Indeed the degree of unisolvence is $m+n+1-d$.

The sets $P_{k}$ are defined by:

$$
P_{m+n+1-2 k}=\left\{a\left|a \in P, \sum_{i=0}^{k}\right| a^{n-i}|+| b^{m-i} \mid>0\right\} .
$$

The second example of a function which is unisolvent of variable degree is $F(a, x)=a_{1} e^{a_{2} x}+a_{3}$ where $P$ is defined by $\left|a_{1}\right|<\infty,\left|a_{2}\right|<\infty,\left|a_{3}\right|<\infty$. When $a_{2}=0$ it is seen that $F$ depends only on $a_{1}+a_{3}$ and $F$ is not solvent of degree 3 in any neighborhood of $F=$ constant. For this example we have $E_{3}-P_{3}=\left\{a \mid a_{2}=0\right\}$ and $P_{2}=E_{3}$. Functions of this form with more terms also come into this class.

The third example of such a such a function is a "restricted polynomial." Let $F=a^{1}+a^{2} x+a^{3} x^{2}$ and $P=\left\{a|| a^{1}|<\infty,| a^{2}|\leqq 10,| a^{3} \mid \leqq 1\right\}$. Then $F$ is unisolvent of degree 3 in the interior of $P$, unisolvent of degree 2 in the interior of the faces of $P$, unisolvent of degree 1 on the edges of $P$.

\section{BIBLIOGRAPHY}

1. N. I. Achieser, Theory of approximation, New York, Frederick Ungar, 1956, Chapter 2.

2. T. S. Motzkin, Approximation by curves of a unisolvent family, Bull. Amer. Math. Soc. vol. 55 (1949) pp. 789-793.

3. - On numerical approximation, Madison, University of Wisconsin Press, 1959, pp. 245-247.

4. L. Tornheim, On n-parameter families of functions and associated convex functions, Trans. Amer. Math. Soc. vol. 69 (1950) pp. 457-467.

The National Bureau of Standards, Washington, District of Columbia

The California Institute of Technology, Pasadena, California 